\section{Commento all'articolo: CKD-MBD: un caso "resistente"}

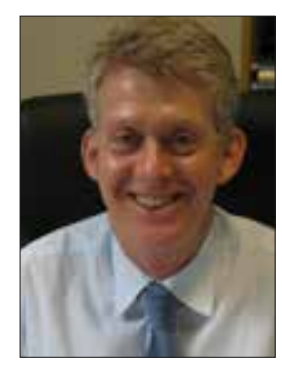

Piergiorgio Messa ria Polidoro (1). terapia oggi disponibile.
Ho aderito con piacere all'invito di aggiungere un mio commento a questo interessante caso molto ben descritto da Ma-

Si tratta di un paziente con insufficienza renale cronica in stadio 5 che presenta una forma di iperparatiroidismo secondario (IPS) particolarmente resistente all'usuale

Alcuni degli aspetti più intriganti sono stati già affrontati in modo efficace ed esaustivo sia da Adriana Dusso e Maria Vittoria Arcidiacono (2) che da Giuseppe Vezzoli et al. (3).

Vorrei limitarmi ad aggiungere alcune brevi considerazioni personali sui tre punti seguenti:

- Resistenza e limiti intrinseci delle terapie disponibili per l'IPS, sia mediche che chirurgiche.

- Deficit di vitamina D naturale e fabbisogno di vitamina D ormonale.

- Come valutiamo il successo della terapia indirizzata al controllo dell'IPS della CKD-MBD.

In alcune nostre recenti pubblicazioni (elencate a fine di questi brevi commenti) abbiamo avuto modo di commentare già su questi punti e rimando pertanto ai riferimenti bibliografici riportati in questi articoli (4-7).

\section{Resistenza e limiti intrinseci delle terapie, mediche e chi- rurgiche}

Nel caso descritto è inconfutabilmente presente una particolare resistenza a dosi inusualmente elevate dei metaboliti attivi di vitamina D (paricalcitolo $45 \mathrm{mcg} /$ settimana): rimando le riflessioni su questo punto al paragrafo seguente, per le ovvie connessioni fisiopatologiche.

Mi soffermerei invece a commentare lo scarso risultato ottenuto con il calciomimetico nel paziente descritto. Di fatto, più che di una resistenza al farmaco, in questo caso si tratta di una limitazione nel suo uso, legata a uno degli effetti intrinseci del farmaco stesso: I'induzione di ipocalcemia. II paziente descritto presentava di fatto, sin dall'inizio della sua storia clinica, bassi valori di calcemia, aggravatisi poi con l'introduzione di cinacalcet se pure a dosi basse (30 mg/die). Questo risultato, per un certo verso atteso, sottolinea come in presenza di ipocalcemia la finestra terapeutica nell'uso del calciomimetico sia notevolmente ridotta. Come spunto di riflessione pratico, se l'percalcemia rappresenta un limite all'uso dei metaboliti attivi della vitamina $D$ è altrettanto vero che l'ipocalcemia, anche relativa e moderata, è spesso un limite nell'uso del calciomimetico.

I limiti non sono però una prerogativa della sola terapia medica. Infatti, anche la soluzione chirurgica dell'IPS ha dei limiti intrinseci. Nel caso descritto, la paratiroidectomia (PTX), sebbene effettuata con una tecnica considerata ottimale (PTX 7/8) è risultata infruttuosa. Questo sottolinea ancora una volta che la PTX nell'IPS presenta tuttora numerose criticità. Per elencare gli aspetti più critici e non definiti:

- $\quad$ non vi è consenso su quali siano le attuali indicazioni nel paziente in dialisi (età, rapporti con il trapianto ecc.);

- quale debba essere la tempistica ideale della PTX nel paziente in lista di trapianto (prima o dopo il trapianto);

- $\quad$ se e quali esami di localizzazione delle ghiandole paratiroidee debbano essere effettuati;

- quale sia la tecnica chirurgica associata a migliori risultati (totale con o senza reimpianto, subtotale $7 / 8$, subtotale con risparmio delle ghiandole apparentemente non iperplastiche, terapie interventistiche di ablazione delle ghiandole con tecniche alterative a quelle chirurgiche).

Inoltre, tutto questo presuppone la presenza di chirurghi esperti in questo tipo di chirurgia e questo punto è divenuto ancor più critico negli ultimi tempi, come conseguenza di una ridotta richiesta di tale tipo di intervento, il che ha reso molto complicato mantenere efficace la curva di apprendimento delle nuove generazioni di chirurghi.

La soluzione potrebbe essere quella di individuare un équipe chirurgica di riferimento che diventi centro di riferimento per questo tipo di attività in un'area interaziendale più vasta.

Rimane comunque implicito che non esiste una terapia per I'IPS migliore in senso assoluto rispetto alle altre. L'utilizzo di tutte le opportunità mediche e chirurgiche oggi disponibili, in vario modo combinate tra di loro, dovrebbe appartenere a un'unica strategia di intervento, con la consapevolezza comunque che siamo lontani dall'avere risolto del tutto il problema del controllo terapeutico dell'IPS.

\section{Deficit di vitamina D naturale e fabbisogno di vitamina D ormonale}

Non vi è dubbio che il paziente descritto ha presentato una chiara resistenza all'effetto dei metaboliti attivi della vitamina D, avendo mantenuto livelli stabilmente elevati di PTH con dosi massive di paracalcitolo ( $45 \mathrm{mcg} / \mathrm{settimana}$ ), senza una sostanziale modificazione dei bassi livelli calcemici. Adriana Dusso e Maria Vittoria Arcidiacono (2) hanno argomentato in modo più che esaustivo su tale argomento. Pertanto mi limiterei ad aggiungere alcune brevi considerazioni che ho avuto occasione di esprimere più volte in altre occasioni.

Una volta che si instauri l'insufficienza renale il deficit della quota ormonale della vitamina $D$ (calcitriolo) è senza dubbio un evento pressoché obbligato. La universale consapevolezza di 
questo fenomeno, la scarsa importanza data sino a non molto tempo fa dai nefrologi alla quota di vitamina $D$ naturale circolante e la convinzione che, una volta fornito il metabolita attivo, non vi fosse alcun bisogno di somministrare il suo precursore hanno portato a ritenere che la terapia del deficit vitaminico $D$ nel paziente nefropatico dovesse essere affrontato con l'utilizzo esclusivo dei metaboliti attivi.

Si sono poi accumulate nel tempo una serie di conoscenze sperimentali e cliniche che hanno fatto rivalutare il ruolo fisiopatologico del deficit della vitamina $D$ naturale in numerosi aspetti clinici connessi non solo con la patologia del metabolismo osseo e minerale. Inoltre, si sono aggiunte evidenze che hanno dimostrato come la cinetica della produzione autocrina e paracrina della vitamina $D$, a livello di numerosi tessuti e organi, abbia meccanismi di controllo omeostatico differenti, quando non opposti, a quelli che regolano la sintesi della vitamina $D$ attiva con funzioni ormonali, prodotta pressoché esclusivamente a livello renale.

Tutto ciò, unito alla crescente consapevolezza dell'elevata frequenza con la quale è presente un deficit di vitamina $D$ naturale nei pazienti nefropatici, ha indotto a utilizzare sempre di più le supplementazioni di vitamina $D$ naturale anche nei pazienti con malattia renale cronica.

Il caso presentato dimostra emblematicamente che solo dopo la correzione del deficit di vitamina $D$ naturale si è ottenuto un netto miglioramento dei parametri metabolici associati all'IPS.

Varrebbe la pena di sottolineare ancora una volta che i due tipi di intervento (supplementazione di vitamina $D$ naturale e di metaboliti attivi) non sono mutualmente escludenti, essendoci a mio parere un razionale nella somministrazione contemporanea di entrambi.

\section{Come valutiamo il successo della terapia indirizzata al controllo dell'IPS della CKD-MBD}

Sarebbe altrettanto opportuno, a mio avviso, ricordare che la misura del "soddisfacimento" del nefrologo riguardo il successo terapeutico nell'IPS del paziente con malattia renale continua a basarsi sostanzialmente sull'entità del controllo dei valori numerici di quei parametri su cui da sempre si basa la diagnosi di tale patologia: livelli ematici di calcio, fosforo e PTH.

Siamo tutti più che consapevoli che questo spesso non basta a mettere al riparo i nostri pazienti da un'evoluzione negativa sia della loro patologia ossea che vascolare (obiettivi primari della terapia della CKD-MBD!), ma di fatto non abbiamo molto di più.

Sappiamo bene che solo la biopsia ossea potrebbe fornirci delle informazioni più valide quantomeno sullo stato osseo, ma conosciamo tutti quanto difficile sia proporre ed effettuare tale accertamento nella maggior parte dei nostri pazienti, ancor più in considerazione del fatto che, perché la biopsia ossea fornisca un valore pienamente informativo, dovrebbe essere eseguita in tempi ripetuti, in relazione agli interventi terapeutici.

Peraltro, le considerazioni fatte a riguardo dell'esecuzione della PTX valgono anche e ancora di più per questo punto di particolare criticità: un numero sempre minore di nefrologi effettua tale tipo di indagine e un ancor minore numero di anatomo-patologi sono in grado di effettuare una lettura affidabile della biopsia ossea (almeno per quelle che sono le informazioni che a noi interessano).

Volendo però terminare questo mio breve commento con una nota di moderato positivismo, non posso non ricordare che abbiamo riacquistato consapevolezza che alcune semplici indagini radiologiche ( $\mathrm{Rx}$ della colonna dorsale e lombare), se pure antiche e poco frequentate, possono ancora darci informazioni importanti e difficilmente confutabili su due outcome clinici di estrema importanza: le fratture vertebrali e le calcificazioni aortiche ...

Probabilmente i nostri pazienti potrebbero essere molto più interessati a conoscere che non hanno più sviluppato fratture ossee e calcificazioni vascolari di quanto non possano esserlo a sapere che i livelli del loro PTH, calcio e fosforo sono rimasti entro intervalli numerici dei quali a loro (e forse talvolta anche a noi !) sfugge il reale significato clinico.

\section{Disclosures}

Financial support: No financial support was received for this submission. Conflict of interest: The author has no conflict of interest with this submission.

\section{Piergiorgio Messa}

Direttore U.O. di Nefrologia, Dialisi e Trapianto Renale, Fondazione Ca' Granda IRCCS Policlinico, Milano

Indirizzo per la corrispondenza:

Prof. Piergiorgio Messa

Direttore UO di Nefrologia, Dialisi e Trapianto Renale

Fondazione Ca' Granda IRCCS Policlinico

20122 Milano

pmessa@policlinico.mi.it

\section{Bibliografia}

1. Polidoro M. CKD-MBD: un caso "resistente". Giornale di Tecniche Nefrologiche e Dialitiche 2014;26:348-58.

2. Dusso A, Arcidiacono MV. La carbamazepina antagonizza la soppressione di Vitamina D-mediata dell'iperparatiroidismo secondario: patogenesi e possibilità terapeutiche. Giornale di Tecniche Nefrologiche e Dialitiche. 2014;26(4):354-58.

3. Vezzoli G, Macrina L, Arcidiacono T. Citocromi P450 ed interazione tra i farmaci. Giornale di Tecniche Nefrologiche e Dialitiche 2014;26:359-60.

4. Messa P, Regalia A, Alfieri CM, Cresseri D, Forzenigo L, Gandolfo MT, Rastaldi MP. Current indications to parathyroidectomy in CKD patients before and after renal transplantation. J Nephrol 2013;26(6):1025-32.

5. Messa P, Alfieri C, Rastaldi MP. Recent insights into vitamin D and its receptor. J Nephrol 2011;24(Suppl 18):S30-7. .

6. Messa P, Alfieri C, Brezzi B. Clinical utilization of cinacalcet in hypercalcemic conditions. Expert Opin Drug Metab Toxicol 2011; 7(4):517-28.

7. Messa P, Alfieri C, Brezzi B. Cinacalcet: pharmacological and clinical aspects. Expert Opin Drug Metab Toxicol 2008;4(12): 1551-60.

Published online: February 4, 2015 